\title{
Strategies for Measurement Error Analysis in Coordinate Measuring Machines
}

F.Z. WILKE, J.B.S de OLIVEIRA, D.M. CLAUDIO, Instituto de Matemática, Faculdade de Informática, Pontifícia Universidade Católica do Rio Grande do Sul, Av. Ipiranga, 6681, 90619-900 Porto Alegre, RS, Brazil.

\section{Introduction}

The quality of products described through precise dimensional characteristics can be verified through careful checking of measurements, taking into account the desired, exact value and the value obtained from direct measurement of the part.

Measures obtained from a CMM (Coordinate Measuring Machine) approximate geometrical features of the object. This instrument, the CMM, as described in [2], makes possible the pointwise metrological recognition by grouping the constituent surfaces of the object.

The measurement of objects is made by coordinate sampling and achieved considering a particular measurement region defined by the displacement system of the mobile parts of the CMM. This measurement is submitted to at least, three kinds of error: systematical, random and rounding error. Unfortunately modeling these errors is a very complex task, as errors are independently related to several external factors that are usually impossible to have under control at all times in an industrial environment.

As error sources cannot all be controlled, it is usually more interesting to analyze the final, net error produced in the measurement. Doing so, and with a relatively simple strategy to analyze the accuracy of measurements obtained from the CMM calibration process, the operator may find the most appropriate region to place a part in order to minimize error taking into account the shape of each part. This system will be presented in the following sections. 


\section{Structure and operation of a CMM}

A Coordinate Measuring Machine is an eletronic and mechanic system built to obtain cartesian coordinates of points on solid surfaces. CMMs differ among themselves but they present common fundamental characteristics. In Figure 1 below we can see the elements that compose a CMM.

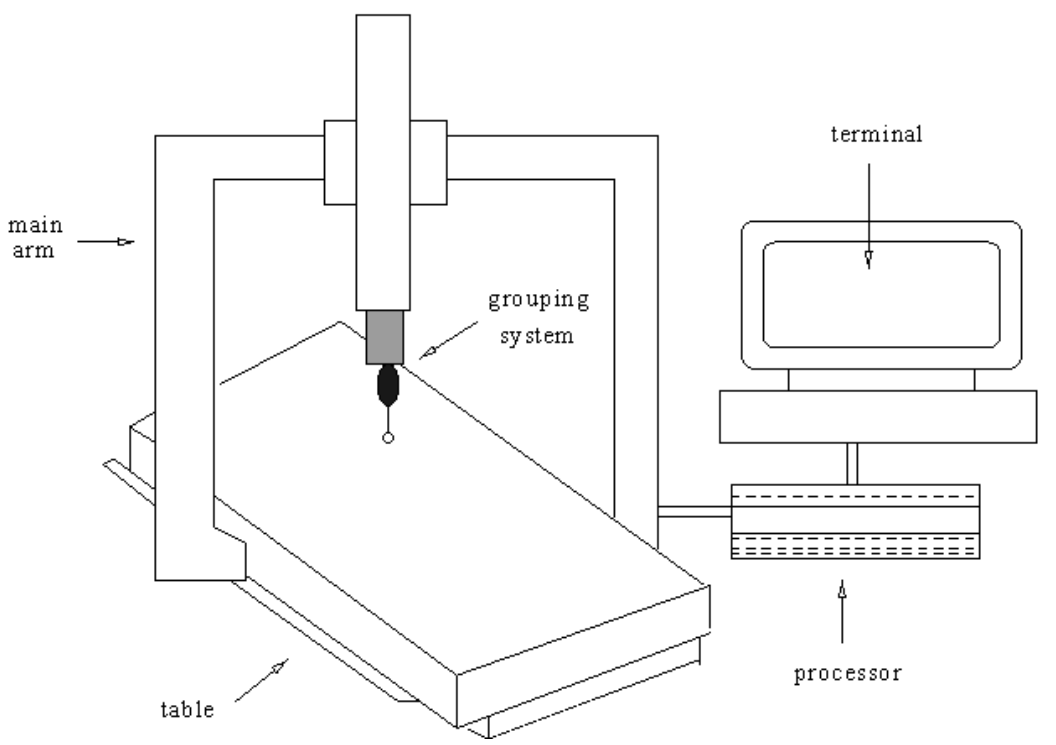

Figure 1. Coordinate Measuring Machine

The grouping system that will touch the object is a very delicate structure coordinates of points placed on a rigid surface when these touch a contact sphere. The function of the grouping system is to transmit a touch event to the processor so that coordinates are noted down and mobile parts are locked. With this system we can distinguish between points less than $0.001 \mathrm{~mm}$ apart from each other, being sufficient for most applications.

\section{Sources of error in a CMM}

Errors in operations with the CMM come basically from the acquisition of the coordinates. The error factors are classified as internal - originating from the machine itself; and external - dependent from environmental conditions. Some of these factors are listed below, and from this list the reader will understand how hard they are to avoid or even to reduce. 
Internal Factors: placement position, coordinate system, algorithms, age of the machine.

External Factors: temperature, environmental humidity, dust, vibrations, human operator.

\section{Errors originating from a CMM}

The errors originating from a CMM can be classified among three types of error: systematical, random and rounding error. These can be described as follows:

1. Systematical error: this error is due to permanent construction imperfections of the instrument and has always the same value at each measurement - thus, it is predictable.

2. Random error: the random error is caused by alterations (not perceptible) of the instrument, of the object to be measured, of the environment and others. These errors cannot be estimated separately but, nevertheless, can be measured quantitatively through calibration.

3. Rounding error: rounding error (see [2]) is inherent to hardware and its control depends on numerical methods and techniques of scientific computing. In this way, we can say that the rounding error is related to processing in finite precision. However, in most cases the rounding error is seldom taken into consideration for practical purposes because the introduced error will be irrelevant if it is compared with the embedded errors of the CMM.

\section{Formulation of the problem}

The measurement of objects by coordinates is achieved considering a particular space, defined by the displacement system of the mobile parts of the machine.

To model this problem, let there be a tridimensional measurement region $S_{p}=\left\{\left(x_{i}, y_{i}, z_{i}\right) / x_{i}, y_{i}, z_{i} \in R, 1 \leq i \leq n\right\}$, where $n$ is the number of positions in the measurement region. We then define error sets originating from $m_{i}$ samples obtained through calibration and associated to each point of $S_{p}$. So, these error sets can be grouped according to each axis:

$$
\begin{aligned}
S_{x}^{i} & =\left\{x_{i}+x_{j}^{i}, 1 \leq i \leq n, 1 \leq j \leq m_{i}\right\} \\
S_{y}^{i} & =\left\{y_{i}+y_{j}^{i}, 1 \leq i \leq n, 1 \leq j \leq m_{i}\right\} \\
S_{z}^{i} & =\left\{z_{i}+z_{j}^{i}, 1 \leq i \leq n, 1 \leq j \leq m_{i}\right\} .
\end{aligned}
$$

That is, for each position $\left(x_{i}, y_{i}, z_{i}\right) \in S_{p}$ are associated three sets of sampled coordinates: $S_{x}^{i}, S_{y}^{i}$ and $S_{z}^{i}$ as shown in the Figure 2 below, for some $i$ and $m_{i}=3$. 


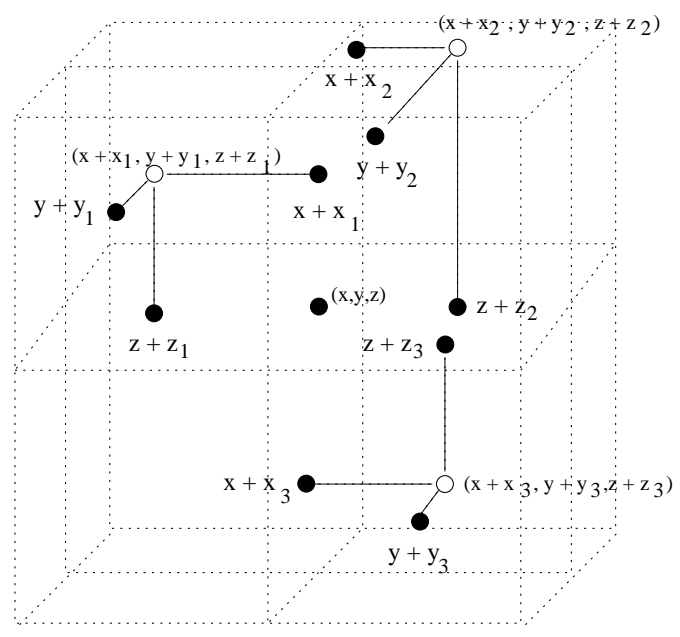

Figure 2. $S_{x}^{i} S_{y}^{i}, S_{z}^{i}$, for a Given Point $i$

Moreover, we introduce a generic error function $\varepsilon_{i}^{k}: R^{3} \times S_{x}^{i} \times S_{y}^{i} \times S_{z}^{i} \longrightarrow R$ to provide error indexes at a position $\left(x_{i}, y_{i}, z_{i}\right)$ taking into account the kind of error we want to measure, the original point and the errors of the measurements obtained.

\section{Proposed solution}

Starting from a data file originated from a calibration containing the original $n$ positions and the $m_{i}$ measurements associated to each position, a set of routines was implemented to calculate the error index associated to each point of the measurement region. This error function associates each point of the measurement space to an error from the $m_{i}$ measurements obtained for the point. So, with these error values we will be able to plot a surface and find the measurement region with smallest error. In this way, errors are associated to a color scale and, finally, a color surface is visualized.

Six different types of error indexes are developed for this analysis, working differently as they allow the user to choose special aspects to take into consideration in the measurement process: sample centering, sample spreading, and others. The program is developed in $\mathrm{C}$ and runs under Windows.

\section{Measurement indexes $\left(\varepsilon_{k}\right)$}

The error value relative to each point of the measurement region will be calculated with six different methods, allowing the user to take into account any several desired aspects. These indexes can be change to suit new applications or special purposes. 


\subsection{Largest absolute error $\left(\varepsilon_{1}\right)$}

Let there be the set $S_{i}$ and the following associates values:

$$
\begin{aligned}
& \nabla x_{i}=\min \left\{x_{j}^{i} / 1 \leq j \leq m_{i}\right\} \triangle x_{i}=\max \left\{x_{j}^{i} / 1 \leq j \leq m_{i}\right\} \\
& \nabla y_{i}=\min \left\{y_{j}^{i} / 1 \leq j \leq m_{i}\right\} \triangle y_{i}=\max \left\{y_{j}^{i} / 1 \leq j \leq m_{i}\right\} \\
& \nabla z_{i}=\min \left\{z_{j}^{i} / 1 \leq j \leq m_{i}\right\} \triangle z_{i}=\max \left\{z_{j}^{i} / 1 \leq j \leq m_{i}\right\} .
\end{aligned}
$$

The measurement index associated to point $\left(x_{i}, y_{i}, z_{i}\right)$ and denoted by $\varepsilon_{1}^{i}$ is evaluated by:

$$
\varepsilon_{1}^{i}=\max \left(\left|\nabla x_{i}\right|,\left|\triangle x_{i}\right|,\left|\nabla y_{i}\right|,\left|\triangle y_{i}\right|,\left|\nabla z_{i}\right|,\left|\triangle z_{i}\right|\right)
$$

This index determines the largest absolute error obtained in the calibration of each point independently of the axes.

\subsection{Largest absolute error + displacement origin-point $\left(\varepsilon_{2}\right)$}

Let there be the set $S_{i}$ and the associated values $\nabla x_{i}, \triangle x_{i}, \nabla y_{i}, \triangle y_{i}, \nabla z_{i}, \triangle z_{i}$ as above.

The measurement index associated to $\left(x_{i}, y_{i}, z_{i}\right)$ and denoted by $\varepsilon_{2}^{i}$ is calculated as:

$$
\varepsilon_{2}^{i}=\max \left(\left|\nabla x_{i}\right|,\left|\triangle x_{i}\right|,\left|\nabla y_{i}\right|,\left|\triangle y_{i}\right|,\left|\nabla z_{i}\right|, \triangle z_{i} \mid \cdot \max \left(x_{i}, y_{i}, z_{i}\right)\right.
$$

This index is similar to the previous but takes into account the distance to the origin. It tries to include the displacement as the CMM will move longer if the object is far from the origin, and the probability of mechanical misaligments increases. Of course, there are other possibilities to represent the displacement in the calculation, and this index can easily be changed to be suited for other applications.

\subsection{Average error of the 3 coordinates $\left(\varepsilon_{3}\right)$}

The measurement index associated to $\left(x_{i}, y_{i}, z_{i}\right)$ denoted by $\varepsilon_{3}^{i}$ is calculated through the formula:

$$
\varepsilon_{3}^{i}=\frac{1}{m_{i}}\left(\sum_{j=1}^{m_{i}} x_{j}^{i}+\sum_{j=1}^{m_{i}} y_{j}^{i}+\sum_{j=1}^{m_{i}} z_{j}^{i}\right)
$$

This index takes the average error of the calibration. We can take just one of the axes (or two) into consideration and create a new index, for example, considering the other axes as irrelevant. 


\subsection{Centering $\left(\varepsilon_{4}\right)$}

Let there be the sets $S_{i}, S_{x}^{i}, S_{y}^{i}$ and $S_{z}^{i}$. The measurement index associated to $\left(x_{i}, y_{i}, z_{i}\right)$ denoted by $\varepsilon_{4}^{i}$ is calculated by:

$$
\varepsilon_{4}^{i}=\frac{1}{m_{i}}\left\|\sum_{i}\left(S_{x}^{i}, S_{y}^{i}, S_{z}^{i}\right)-S_{i}\right\|
$$

This index shows the degree of centering of the samples with respect to $\left(x_{i}, y_{i}, z_{i}\right)$ (see Figure 3).

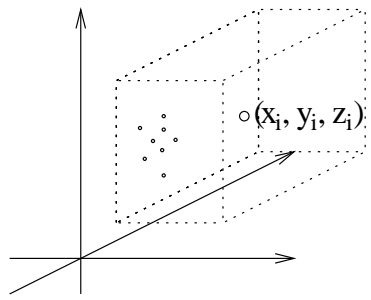

Error index is large

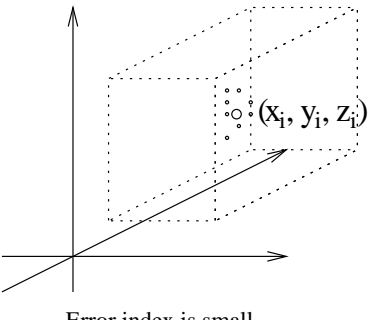

Error index is small

Figure 3. Centering Index

The more distant of the point are the samples, the largest will be the value of this index. If the centering is correct, the center of the samples coincides with $\left(x_{i}, y_{i}, z_{i}\right)$.

\subsection{Spreading from the ideal point $\left(\varepsilon_{5}\right)$}

This index shows the degree of spreading of the samples regarding the ideal point (see Figure 4), denoted by $\varepsilon_{5}^{i}$ and calculated through the formula:

$$
\varepsilon_{5}^{i}=\frac{1}{m_{i}} \sum_{i}\left\|\left(S_{x}^{i}, S_{y}^{i}, S_{z}^{i}\right)-S_{i}\right\| .
$$

The more spreaded from the ideal point are the samples, the largest will be the value of this index.

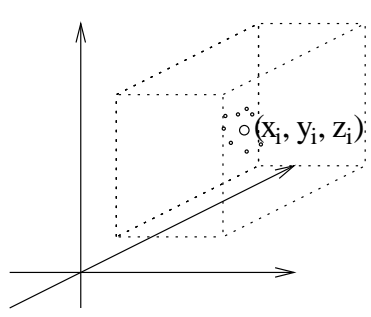

Error index is small

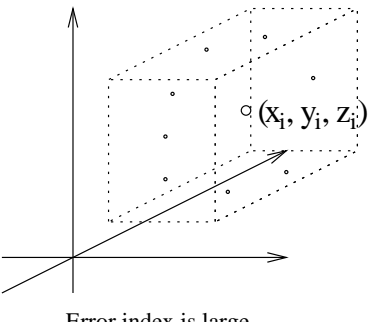

Error index is large

Figure 4. Spreading the Ideal Point 


\subsection{Spreading of the center of the samples $\left(\varepsilon_{6}\right)$}

This index shows the degree of spreading of the errors regarding the center of the samples (se Figure 5) and is associated to $\left(x_{i}, y_{i}, z_{i}\right)$, denoted by $\varepsilon_{6}^{i}$ and calculated through the formula:

$$
\varepsilon_{6}^{i}=\frac{1}{m_{i}} \sum_{i}\left\|\left(S_{x}^{i}, S_{y}^{i}, S_{z}^{i}\right)-C\right\|
$$

where

$$
C=\frac{1}{m_{i}} \sum_{i}\left(S_{x}^{i}, S_{y}^{i}, S_{z}^{i}\right)
$$

The more spreaded from the center of the samples are the samples, the largest will be the value of this index.

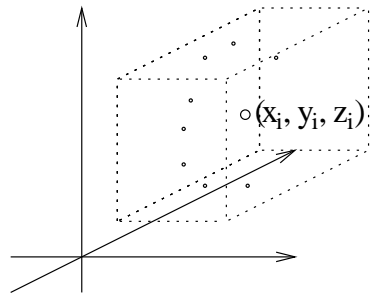

Error index is small

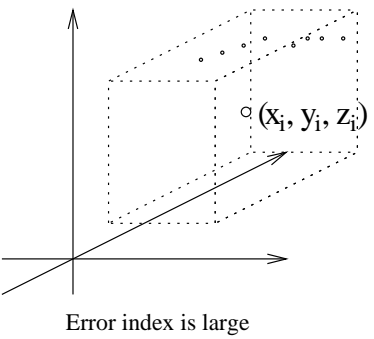

Error index is large

Figure 5. Spreading of the Center of the Samples

Using the visualization tool (

- Schroeder 1996) the indexes are associated to a color scale and color contours of surfaces are shown. The number of contours and their color can be controlled by the user, helping to enhance detail and search for placement positions where error is reduced, or simply to check how error patterns evolve over time.

\section{Interpolation}

To improve and increase the degree of detail of the final image generated by the visualization toolkit (vtk) and due to the small number of points provided by calibration, interpolation is used to generate more intermediate points between the index values associated to each point of the measurement region.

Consider the measurement grid $5 \times 5 \times 5$ (Figure 6) and the index $\left(\varepsilon_{k}^{i}\right)$ associated to each one of the 125 points. 


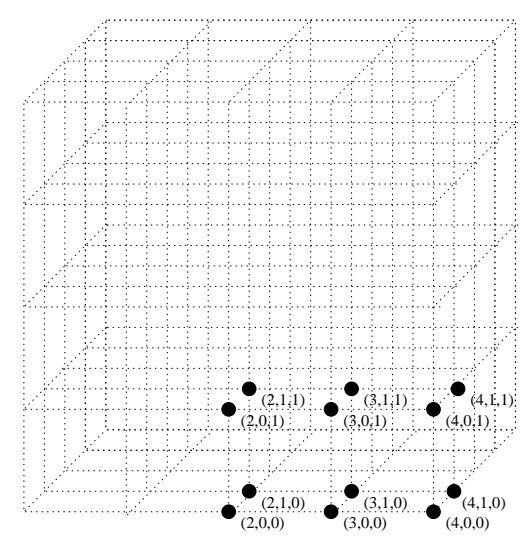

Figure 6. Measurement Grid Considered $(5 \times 5 \times 5)$

More points are defined in this region generating a new grid $(9 \times 9 \times 9)$, for example. The evaluation of the index value associated to the new points generated is made as follows (Figure 7):
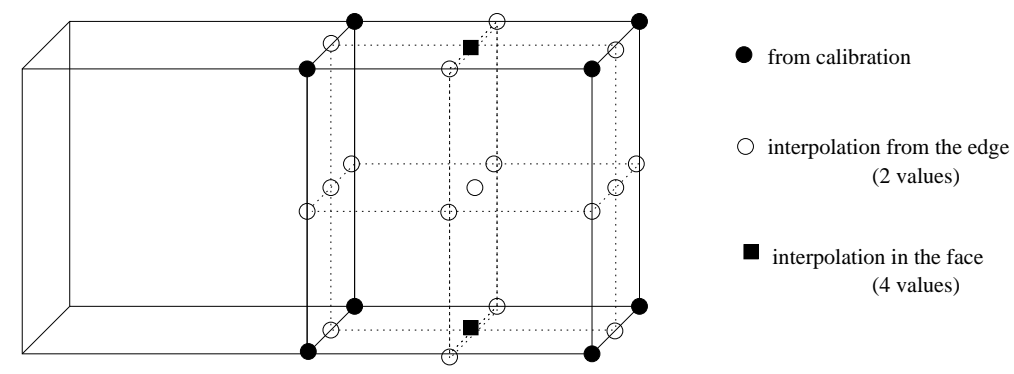

Figure 7. Points Generated Through Interpolation

\section{The interface}

The interface is divided in two parts: Visualization and Edition of colors. It presents a fixed menu with four options: File, Index, Interpolation and Contours.

In the option "File", we can open the input data file. The format of this file is the following (see Figure 8): 


$\begin{array}{rrrrrrrrr}\text { x } & 2 & 0 & 0 & -0.345421 & -0.419010 & -0.401535 & -0.360107 & -0.410598 \\ \text { y } & 2 & 0 & 0 & -0.374845 & -0.375820 & -0.345422 & -0.391471 & -0.418082 \\ \text { z } & 2 & 0 & 0 & -0.427808 & -0.428414 & -0.432152 & -0.426594 & -0.434873 \\ \text { x } & 3 & 0 & 0 & 0.366134 & 0.375101 & 0.352256 & 0.409748 & 0.413160 \\ \text { y } & 3 & 0 & 0 & 0.354491 & 0.430227 & 0.389085 & 0.340028 & 0.420292 \\ \text { z } & 3 & 0 & 0 & 0.427436 & 0.437159 & 0.373964 & 0.363934 & 0.407025\end{array}$

Figure 8. Input Data File

Each axis of the coordinates has five measurements (taken directly from the calibration).

\subsection{Visualization}

The visualization part is composed by three options: Index, Interpolation and Contours.

Under "Index" we can choose by one of the six indexes presented: Largest absolute error, Largest absolute error + displacement origin-point, Average error of the 3 coordinates, Centering, Spreading of the ideal point, Spreading of the center of the samples.

Under "Interpolation" we can choose one of the four measurement spaces presented: $5 \times 5 \times 5,9 \times 9 \times 9,17 \times 17 \times 17,33 \times 33 \times 33$ samples.

Under "Contours", the user can choose by the number of contours in the visualization: $4,6,8,10,12$ or others.

\subsection{Color edition}

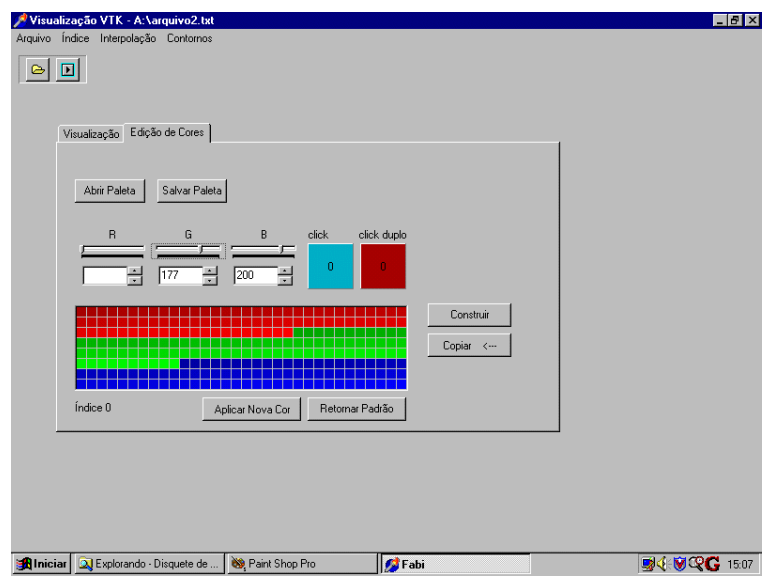

Figure 9. Color Edition

The colors to be used are defined in the window shown in Figure 9. This is made through the manipulation of a color palette presented in the screen. The first cell 
of the palette indicates the color related to value of smallest error, and from here the user has the following options:

- open a palette;

- work with the default palette;

- modify the colors of the default palette;

- return to default palette.

To plot new images is necessary to click in the button depicted as a small arrow to the right (see example of visualization below - Figure 10).

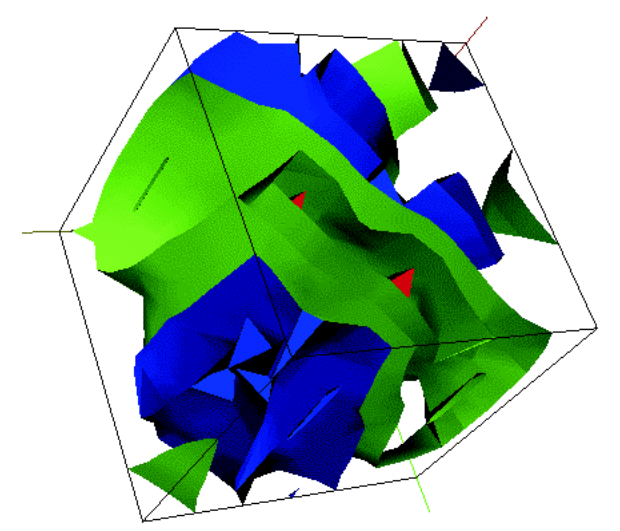

Figure 10. Image

\section{Example}

Considering one input data file containing test data, the measurement index "Average error of the 3 coordinates", the measurement space $5 \times 5 \times 5,4$ contours using the default palette, the following image was obtained (Figure 11): 


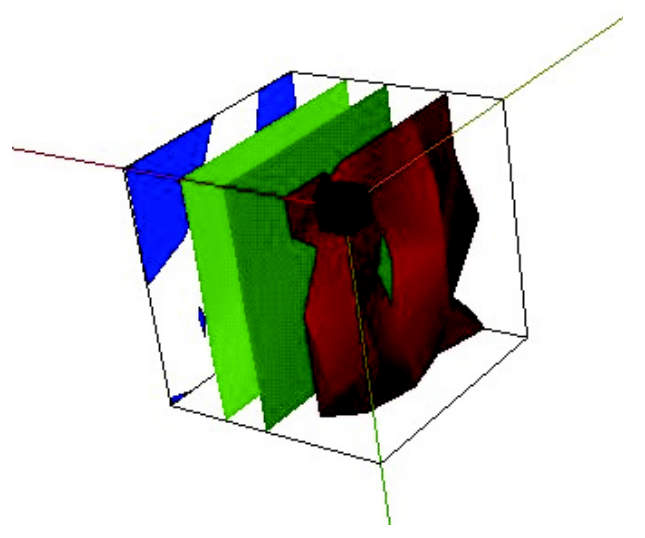

Figure 11. Example

The axes $x, y$ and $z$ are represented in violet, green and blue, respectively. The small cube shows the origin of the system and the initial position of the grouping system.

In this example we have that the green region represents a region of small error and the violet region a region of large error. Thus, it is advisable to place any object to be measure in the green region, since the average error is smaller there.

\section{Conclusions}

This paper deals with the practical issues of using computers in the process of placing objects to be measured by a coordinate measuring machine and to produce better results, reducing overal error.

Calibration data are used to produce tridimensional error maps. Using such maps, the operator may choose the best position to place a part according to its shape and the error that has to be minimized. Moreover, one may produce different error maps for different purposes and keep the "history" of the CMM recorded as a series of error maps taken from periodical calibrations. We could perform comparisons between different machines and automate the process of positioning a part to minimize error.

According to [7], scientific visualization is the formal name given to the field of computer science that encompasses user interface, data representation and processing algorithms, visual representations, and other sensory presentations such as sound or touch. In this sense, this paper presents a graphical environment design oriented to the scientific visualization of the error embedded in the measurement space of a CMM. 


\section{References}

[1] A.S. Alves, "Metrologia Geométrica", Faculdade de Ciência e Tecnologia, Coimbra, Portugal, 1996.

[2] J.A. Bosch, "Coordinate Measuring Machines and Systems", Marcel Dekker, Inc., New York, Basel, 1995.

[3] CMAA, Accuracy Specification for Coordinate Measuring Machines, London, 1989.

[4] ISO 10360-2, Coordinate Metrology, Part 2. Performance assessment of coordinate measuring machines, CH-1211, Geneve 20, Switzerland, 1994.

[5] U. Kulisch and W.L. Miranker, Computer Arithmetic in Theory and Practice, New York, Academic Press, 1981.

[6] H. Kunzmann, E. Trapet and F. Wäldele, Koordinatenmessgeräte in der Kalibrierkette. Physikalisch-Technische Bundesanstalt (PTB)-Mitteilungen 104, 5/94, s.348-356, Braunschweig.

[7] W. Schroeder, K. Martin and B. Lorensen, "The Visualization Toolkit: An Object-oriented Approach to 3D Graphics", Prentice Hall, Upper Saddle River, New Jersey, 1996.

[8] E. Trapet and F. Wäldele, "A Reference Object Based Method to Determine the Parametric Error Components of Coordinate Measuring Machines and Machines Tools", Measurement , 1991.

[9] VDI/VDE 2617, Genauigkeit von Koordinatenmessgeräte, Koengrösssen und deren Prüfung, VDI - Verlag, Düsseldorf, 1986-1991. 